\title{
ON APPROXIMATELY CONVEX TAKAGI TYPE FUNCTIONS
}

\author{
JUDIT MAKÓ AND ZSOLT PÁLES
}

(Communicated by Sergei K. Suslov)

Abstract. Given a nonnegative function $\phi:\left[0, \frac{1}{2}\right] \rightarrow \mathbb{R}_{+}$, we define the Takagi type function $S_{\phi}: \mathbb{R} \rightarrow \mathbb{R}$ by

$$
S_{\phi}(x):=\sum_{n=0}^{\infty} 2 \phi\left(\frac{1}{2^{n+1}}\right) d_{Z}\left(2^{n} x\right),
$$

where $d_{\mathbb{Z}}(x):=\operatorname{dist}(x, \mathbb{Z}):=\inf \{|x-k|: k \in \mathbb{Z}\}$. The main result of the paper states that if $\phi(0)=0$ and the mapping $x \mapsto \phi(x) / x$ is concave, then the Takagi type function $S_{\phi}$ is approximately Jensen convex in the following sense:

$$
S_{\phi}\left(\frac{x+y}{2}\right) \leq \frac{S_{\phi}(x)+S_{\phi}(y)}{2}+\phi \circ d_{\mathbb{Z}}\left(\frac{x-y}{2}\right) \quad(x, y \in \mathbb{R}) .
$$

Applications to the theory of approximately convex functions are also given.

\section{INTRODUCTION}

Throughout this paper denote by $\mathbb{R}, \mathbb{R}_{+}, \mathbb{Z}$, and $\mathbb{N}$ the sets of real numbers, nonnegative real numbers, integers, and positive integers, respectively, and denote by $I$ a nonempty subinterval of $\mathbb{R}$.

Define, for every $x \in \mathbb{R}$,

$$
T(x):=\sum_{n=0}^{\infty} \frac{d_{\mathbb{Z}}\left(2^{n} x\right)}{2^{n}},
$$

where

$$
d_{\mathbb{Z}}(x):=\operatorname{dist}(x, \mathbb{Z}):=\inf \{|x-k|: k \in \mathbb{Z}\} .
$$

The function $T$ is 1-periodic, continuous and vanishes on $\mathbb{Z}$ and it is a well-known example of a continuous but nowhere differentiable real function, which is usually called "van der Waerden's function" (cf. [26]). As was discovered by Knopp [12], this function had been constructed (in terms of the dyadic expansion of $x$ ) almost 30 years before by T. Takagi in 1903 25]. For further historical details and remarks, we refer to the papers by Billingsley [2], Cater [5], and Kairies [11.

Received by the editors March 23, 2010 and, in revised form, October 4, 2011.

2010 Mathematics Subject Classification. Primary 39B62, 26B25.

Key words and phrases. Approximate Jensen convexity, $\phi$-Jensen convexity, Takagi type function.

This research has been supported by the Hungarian Scientific Research Fund (OTKA) Grant NK81402 and by the TÁMOP 4.2.1/B-09/1/KONV-2010-0007 and 4.2.2/B-10/1-2010-0024 projects implemented through the New Hungary Development Plan co-financed by the European Social Fund and the European Regional Development Fund. 
The development of the theory of approximately convex functions began with the paper by Hyers and Ulam [6], who in the year 1952 introduced and investigated $\varepsilon$-convex functions. The Hyers and Ulam decomposition theorem of $\varepsilon$-convex functions was later generalized by Páles in 17. Since then many papers on this subject have been published. An important issue in these papers is to obtain estimations of the bounds which appear for approximately convex functions; see, for example, Cannarsa and Sinestrari [4, Ng and Nikodem [16], Rolewicz [18, 19, 20]. The research and results in this paper are motivated by the fact that Takagi-like functions appear naturally in the investigation of approximate convexity. For details, we recommend the papers by Házy [7, Házy and Páles [8, 9, 10, Makó and Páles [13, 14, Mureńko, Tabor and Tabor [15], Tabor and Tabor [21, 22], Tabor, Tabor, and Żołdak [24, 23].

Let $I$ be a nonempty real interval of $\mathbb{R}$ and denote $I^{*}:=(I-I) \cap \mathbb{R}_{+}$. Let $\varphi: \frac{1}{2} I^{*} \rightarrow \mathbb{R}_{+}$be a given function. A function $f: I \rightarrow \mathbb{R}$ is called $\varphi$-Jensen convex on $I$ (cf. Makó and Páles [13], Tabor and Tabor [21, 22]) if, for all $x, y \in I$,

$$
f\left(\frac{x+y}{2}\right) \leq \frac{f(x)+f(y)}{2}+\varphi\left(\left|\frac{x-y}{2}\right|\right)
$$

If $\varphi(t)=\varepsilon t^{p}\left(t \in \mathbb{R}_{+}\right)$, where $\varepsilon, p$ are nonnegative constants, then $\varphi$-Jensen convex functions are called $(\varepsilon, p)$-Jensen convex functions (cf. [9]).

For a fixed error function $\varphi: \frac{1}{2} I^{*} \rightarrow \mathbb{R}_{+}$, we introduce two Takagi type functions $\mathcal{T}_{\varphi}: \mathbb{R} \times I^{*} \rightarrow \mathbb{R}_{+}$and $\mathcal{S}_{\varphi}: \mathbb{R} \times I^{*} \rightarrow \mathbb{R}_{+}$by

$$
\begin{array}{ll}
\mathcal{T}_{\varphi}(t, u):=\sum_{n=0}^{\infty} \frac{\varphi\left(d_{\mathbb{Z}}\left(2^{n} t\right) u\right)}{2^{n}}, & \left((t, u) \in \mathbb{R} \times I^{*}\right) . \\
\mathcal{S}_{\varphi}(t, u):=\sum_{n=0}^{\infty} 2 \varphi\left(\frac{u}{2^{n+1}}\right) d_{\mathbb{Z}}\left(2^{n} t\right), &
\end{array}
$$

Note that the first series converges uniformly if $\varphi$ is bounded; on the other hand, for the uniform convergence of the second series, it is sufficient if $\sum_{n=n_{0}}^{\infty} \varphi\left(2^{-n}\right)<$ $\infty$ for some $n_{0} \in \mathbb{N}$.

The importance of the function $\mathcal{T}_{\varphi}$ introduced above is enlightened by the following result (cf. [13], 21, 22]), which is a generalization of the celebrated BernsteinDoetsch theorem 1 .

Theorem A. Let $f: I \rightarrow \mathbb{R}$ be locally bounded from above on $I$ (i.e., $f$ is bounded from above on any compact subset of $I$ ) and let $\varphi: \frac{1}{2} I^{*} \rightarrow \mathbb{R}_{+}$. Then $f$ is $\varphi$-Jensen convex on $I$; i.e., (1) holds for all $x, y \in I$ if and only if

$$
f(t x+(1-t) y) \leq t f(x)+(1-t) f(y)+\mathcal{T}_{\varphi}(t,|x-y|)
$$

for all $x, y \in I$ and $t \in[0,1]$.

The other Takagi type function $\mathcal{S}_{\varphi}$ was introduced by Tabor and Tabor. Its role and importance in the theory of approximate convexity is shown by the next theorem $(21])$. 

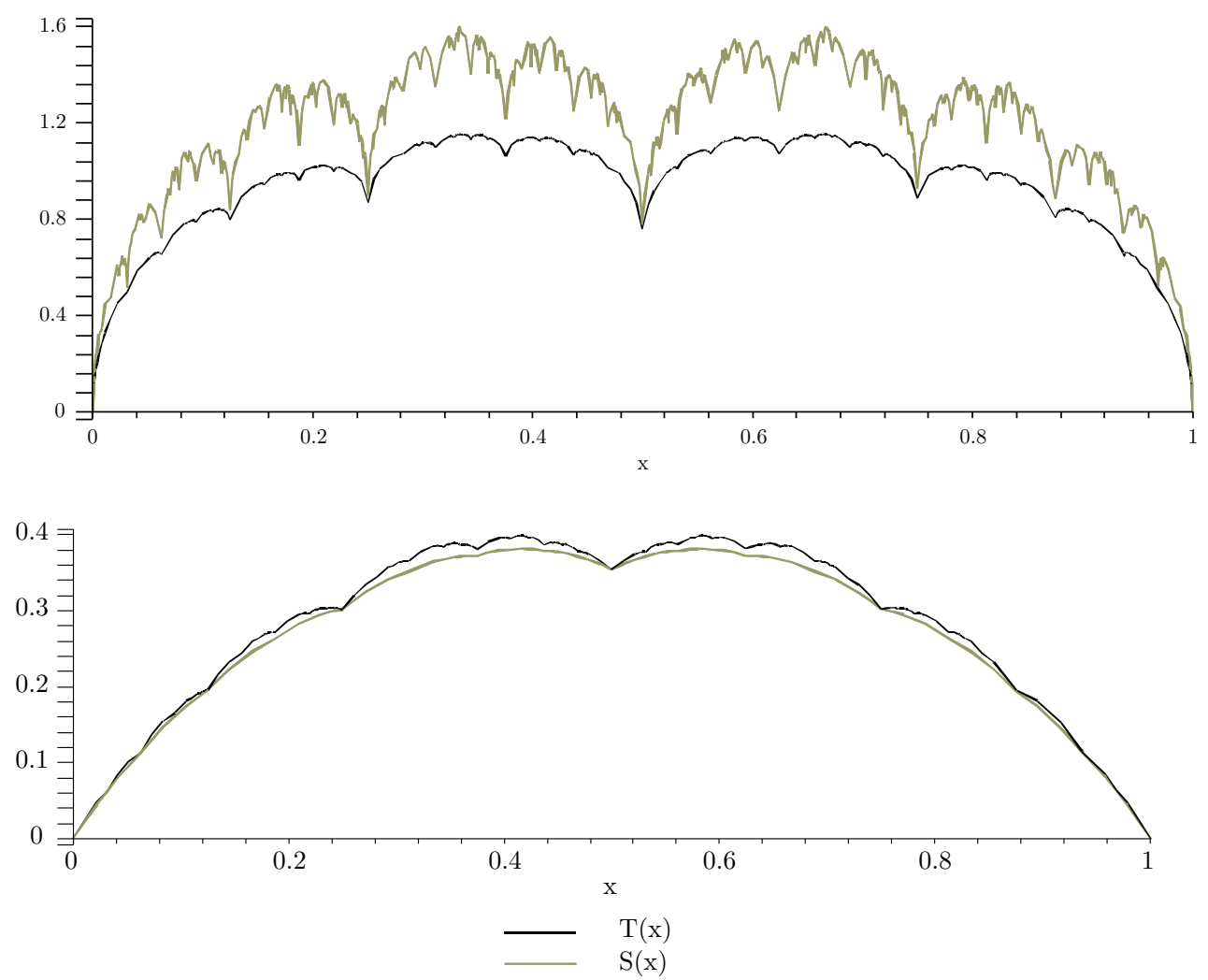

Figure 1

Theorem B. Let $f: I \rightarrow \mathbb{R}$ be locally bounded from above on $I$ (i.e., $f$ is bounded from above on any compact subset of I) and let $\varphi: \frac{1}{2} I^{*} \rightarrow \mathbb{R}_{+}$such that $\sum_{n=n_{0}}^{\infty} \varphi\left(2^{-n}\right)<\infty$ for some $n_{0} \in \mathbb{N}$. Then $f$ is $\varphi$-Jensen convex on $I$; i.e., (11) holds for all $x, y \in I$ if and only if

$$
f(t x+(1-t) y) \leq t f(x)+(1-t) f(y)+\mathcal{S}_{\varphi}(t,|x-y|)
$$

for all $x, y \in I$ and $t \in[0,1]$.

Let $\varepsilon, p \geq 0$ be arbitrary constants. When $\varphi(t)=\varepsilon t^{p}\left(t \in \mathbb{R}_{+}\right)$, the two corollaries below (see [9] and 21]) are immediately consequences of the previous theorems.

For $p>0$, define the Takagi type functions $S_{p}$ and $T_{p}$ by

$$
T_{p}(x):=\sum_{n=0}^{\infty} \frac{\left(d_{\mathbb{Z}}\left(2^{n} x\right)\right)^{p}}{2^{n}}, \quad S_{p}(x):=\sum_{n=0}^{\infty} \frac{d_{\mathbb{Z}}\left(2^{n} x\right)}{2^{n p+p-1}} \quad(x \in \mathbb{R}) .
$$

They generalize the classical Takagi function $T$ in two ways, because $T_{1}=S_{1}=T$ holds obviously. It is less trivial, but it can be proved that $T_{2}(x)=S_{2}(x)=x(1-x)$ for $x \in[0,1]$. Moreover, $T_{p} \leq S_{p}$ holds for $p \in[0,1] \cup[2, \infty[$ and the inequality is reversed for $p \in[1,2]$. The pictures in Figure 1 demonstrate the comparison between $T_{p}$ and $S_{p}$ for $p=0.5$ and $p=1.5$, respectively. 
Corollary A. Let $f: I \rightarrow \mathbb{R}$ be locally bounded from above on $I$ and let $\epsilon, p \geq 0$. Then $f$ is $(\epsilon, p)$-Jensen convex on $I$ if and only if

$$
f(t x+(1-t) y) \leq t f(x)+(1-t) f(y)+\varepsilon T_{p}(t)|x-y|^{p}
$$

for all $x, y \in I$ and $t \in[0,1]$.

Corollary B. Let $f: I \rightarrow \mathbb{R}$ be locally bounded from above on $I$ and let $\epsilon, p \geq 0$. Then $f$ is $(\epsilon, p)$-Jensen convex on $I$ if and only if

$$
f(t x+(1-t) y) \leq t f(x)+(1-t) f(y)+\varepsilon S_{p}(t)|x-y|^{p}
$$

for all $x, y \in I$ and $t \in[0,1]$.

In [3] Boros proved that if $p=1$ and $t \in[0,1]$ is fixed, then $S_{1}(t)=T_{1}(t)=T(t)$ is the smallest possible. In 22 Tabor and Tabor showed that if $1 \leq p \leq 2$ and $t \in[0,1]$ is fixed, then $S_{p}(t)$ is the smallest possible value so that (17) is valid for all $(\epsilon, p)$-Jensen convex functions $f$ on $I$.

In [13] the authors proved the error terms $\mathcal{T}_{\varphi}(t,|x-y|)$ in (3) under certain convexity-monotonicity assumptions on the error function $\varphi$ and also $T_{p}(t)$ in (6) when $0<p \leq 1$ are the smallest possible ones. In other words, the following results provide exact upper bound for the convexity-difference of $\varphi$-Jensen convex functions defined by

(8)

$$
C_{\varphi}(x, y, t):=\sup _{f \in \mathcal{J}_{\varphi}(I)}\{f(t x+(1-t) y)-t f(x)-(1-t) f(y)\} \quad(x, y \in I, t \in[0,1]),
$$

where

$\mathcal{J C}_{\varphi}(I):=\{f: I \rightarrow \mathbb{R} \mid f$ is locally bounded from above and $\varphi$-Jensen convex on $I\}$.

Theorem C. Let $\varphi: \frac{1}{2} I^{*} \rightarrow \mathbb{R}_{+}$be a differentiable function with $\varphi(0)=0$ such that $\varphi$ is nondecreasing, concave and $\varphi^{\prime}$ is convex on $\frac{1}{2} I^{*}$. Then, for all $x, y \in I$ and $t \in[0,1]$,

$$
C_{\varphi}(x, y, t)=\mathcal{T}_{\varphi}(t,|x-y|) .
$$

Corollary C. Let $0<p \leq 1$ and $\varepsilon \geq 0$. Let $\varphi_{p}: \mathbb{R}_{+} \rightarrow \mathbb{R}_{+}$be defined by $\varphi_{p}(t):=\varepsilon t^{p}\left(t \in \mathbb{R}_{+}\right)$. Then, for all $x, y \in I$ and $t \in[0,1]$,

$$
C_{\varphi_{p}}(x, y, t)=\varepsilon T_{p}(t)|x-y|^{p} .
$$

The main purpose of this paper is to prove that the inequality (41) is also sharp under a certain concavity assumption on $\varphi$.

To approach and discuss this problem, we introduce the Takagi type function $S_{\phi}: \mathbb{R} \rightarrow \mathbb{R}$ defined by

$$
S_{\phi}(x)=\sum_{n=0}^{\infty} 2 \phi\left(\frac{1}{2^{n+1}}\right) d_{\mathbb{Z}}\left(2^{n} x\right),
$$

where $P:=\left\{\frac{1}{2}, \frac{1}{4}, \ldots, \frac{1}{2^{n}}, \ldots,\right\}$ and $\phi: P \rightarrow \mathbb{R}_{+}$is a nonnegative function. Taking $\phi(t):=t^{p}\left(t \in \mathbb{R}_{+}\right)$, we obtain the function $S_{p}$ defined in (5).

In Theorem 4 and Theorem 9 of Section 2 below, we will prove that for a certain class of functions $\phi$, the Takagi type function $S_{\phi}$ is approximately Jensen convex. In Section 3, we shall apply this result to obtain the sharpness of the error term in Theorem B. 


\section{Approximate convexity of TAKagi type Functions}

The main results of this section state that under certain assumptions on the function $\phi: P \rightarrow \mathbb{R}$ (where $P:=\left\{\frac{1}{2}, \frac{1}{4}, \ldots, \frac{1}{2^{n}}, \ldots,\right\}$ ), $S_{\phi}$ is well-defined and approximately Jensen convex in the following sense: For all $x, y \in \mathbb{R}$,

$$
S_{\phi}\left(\frac{x+y}{2}\right) \leq \frac{S_{\phi}(x)+S_{\phi}(y)}{2}+\phi \circ d_{\mathbb{Z}}\left(\frac{x-y}{2}\right) .
$$

First we describe the situation when the definition of $S_{\phi}$ is correct.

Lemma 1. Let $\phi: P \rightarrow \mathbb{R}_{+}$be a nonnegative function. Then $S_{\phi}$ is well-defined; i.e., the series on the right-hand side of (9) is convergent everywhere if and only if

$$
\sum_{n=1}^{\infty} \phi\left(\frac{1}{2^{n}}\right)<\infty
$$

Proof. Observe that $d_{\mathbb{Z}}\left(2^{n} \frac{1}{3}\right)=\frac{1}{3}$ for all $n \in \mathbb{N}$. Hence the convergence of the right-hand side of (9) at $x=\frac{1}{3}$ yields that (10) holds.

Conversely, if (10) is satisfied, then, for all $x \in \mathbb{R}$,

$$
S_{\phi}(x)=\sum_{n=0}^{\infty} 2 \phi\left(\frac{1}{2^{n+1}}\right) d_{\mathbb{Z}}\left(2^{n} x\right) \leq \sum_{n=0}^{\infty} \phi\left(\frac{1}{2^{n+1}}\right)<\infty,
$$

which proves the statement.

In the sequel, the class of nonnegative functions $\phi: P \rightarrow \mathbb{R}_{+}$satisfying the condition (10) will be denoted by $\mathcal{H}$ :

$$
\mathcal{H}:=\left\{\phi: P \rightarrow \mathbb{R}_{+} \mid \sum_{n=1}^{\infty} \phi\left(\frac{1}{2^{n}}\right)<\infty\right\} .
$$

The next theorem, which was discovered by Jacek Tabor and Józef Tabor, has an important role in the proof of our main theorem.

Theorem 2 ([22]). For every $p \in[1,2]$ and $x, y \in \mathbb{R}$,

$$
S_{p}\left(\frac{x+y}{2}\right) \leq \frac{S_{p}(x)+S_{p}(y)}{2}+d_{\mathbb{Z}}^{p}\left(\frac{x-y}{2}\right) .
$$

In the next result we give a representation of $S_{\phi}(x)$ as an infinite linear combination of the values $S_{p}\left(2^{n} x\right), n=1,2, \ldots$ The particular case when $p=2$ and $\phi(x)=x^{q}$ for some $q \in[1,2]$ was established in [22].

Theorem 3. Let $\phi \in \mathcal{H}$. Then, for every $p>0$ and $x \in \mathbb{R}$,

$$
S_{\phi}(x)=2^{p} \phi\left(\frac{1}{2}\right) S_{p}(x)+\sum_{n=1}^{\infty}\left(2^{p} \phi\left(\frac{1}{2^{n+1}}\right)-\phi\left(\frac{1}{2^{n}}\right)\right) S_{p}\left(2^{n} x\right) .
$$

Proof. Since $S_{p}(x)=\sum_{n=0}^{\infty} \frac{1}{2^{n p+p-1}} d_{\mathbb{Z}}\left(2^{n} x\right)$, the right-hand side of (11) can be written in the form $\sum_{n=0}^{\infty} a_{n} d_{\mathbb{Z}}\left(2^{n} x\right)$, where

$$
\begin{aligned}
a_{n} & =2^{p} \phi\left(\frac{1}{2}\right) \frac{1}{2^{n p+p-1}}+\sum_{k=1}^{n} \frac{1}{2^{(n-k) p+p-1}}\left(2^{p} \phi\left(\frac{1}{2^{k+1}}\right)-\phi\left(\frac{1}{2^{k}}\right)\right) \\
& =\phi\left(\frac{1}{2}\right) \frac{1}{2^{n p-1}}+\sum_{k=1}^{n}\left(\frac{1}{2^{(n-k) p-1}} \phi\left(\frac{1}{2^{k+1}}\right)-\frac{1}{2^{(n-k+1) p-1}} \phi\left(\frac{1}{2^{k}}\right)\right)=2 \phi\left(\frac{1}{2^{n+1}}\right) .
\end{aligned}
$$

Consequently (11) holds. 
An immediate consequence of the previous two theorems is the next result, which states the approximate convexity of $S_{\phi}$.

Theorem 4. Let $\phi \in \mathcal{H}$ such that, for all $u \in \frac{1}{2} P, \phi(2 u) \leq 2^{p} \phi(u)$, where $p \in[1,2]$ is an arbitrary constant. Then, for all $x, y \in \mathbb{R}$,

$$
S_{\phi}\left(\frac{x+y}{2}\right) \leq \frac{S_{\phi}(x)+S_{\phi}(y)}{2}+\Phi_{p}\left(\frac{x-y}{2}\right),
$$

where $\Phi_{p}: \mathbb{R} \rightarrow \mathbb{R}$ is defined by

$$
\Phi_{p}(u):=\sum_{n=0}^{\infty} \phi\left(\frac{1}{2^{n+1}}\right)\left(2^{p} d_{\mathbb{Z}}^{p}\left(2^{n} u\right)-d_{\mathbb{Z}}^{p}\left(2^{n+1} u\right)\right) .
$$

Proof. For an arbitrary function $f: \mathbb{R} \rightarrow \mathbb{R}$ define $J f: \mathbb{R}^{2} \rightarrow \mathbb{R}$ by

$$
J f(x, y):=f\left(\frac{x+y}{2}\right)-\frac{f(x)+f(y)}{2} .
$$

Then, by (11), we get

$$
J S_{\phi}(x, y)=2^{p} \phi\left(\frac{1}{2}\right) J S_{p}(x, y)+\sum_{n=1}^{\infty}\left(2^{p} \phi\left(\frac{1}{2^{n+1}}\right)-\phi\left(\frac{1}{2^{n}}\right)\right) J S_{p}\left(2^{n} x, 2^{n} y\right) .
$$

By Theorem 2, for all $x, y \in \mathbb{R}$, we have that

$$
J S_{p}(x, y) \leq d_{\mathbb{Z}}\left(\frac{x-y}{2}\right)^{p} .
$$

On the other hand, by the assumption on $\phi$, the coefficient $\left(2^{p} \phi\left(\frac{1}{2^{n+1}}\right)-\phi\left(\frac{1}{2^{n}}\right)\right)$ is nonnegative; hence for all $x, y \in \mathbb{R}$, we get

$$
\begin{aligned}
J S_{\phi}(x, y) & \leq 2^{p} \phi\left(\frac{1}{2}\right) d_{\mathbb{Z}}^{p}\left(\frac{x-y}{2}\right)+\sum_{n=1}^{\infty}\left(2^{p} \phi\left(\frac{1}{2^{n+1}}\right)-\phi\left(\frac{1}{2^{n}}\right)\right) d_{\mathbb{Z}}^{p}\left(\frac{2^{n}(x-y)}{2}\right) \\
& =\sum_{n=0}^{\infty} \phi\left(\frac{1}{2^{n+1}}\right)\left(2^{p} d_{\mathbb{Z}}^{p}\left(\frac{2^{n}(x-y)}{2}\right)-d_{\mathbb{Z}}^{p}\left(\frac{2^{n+1}(x-y)}{2}\right)\right)=\Phi_{p}\left(\frac{x-y}{2}\right),
\end{aligned}
$$

which proves (12).

In the next proposition we describe a decomposition property of the function $\Phi_{p}$.

Proposition 5. Let $\phi \in \mathcal{H}$ and $p \in[1,2]$. Then, for all $\left.u \in] 0, \frac{1}{2}\right]$,

$$
\Phi_{p}(u)=\Phi_{p}\left(\frac{1}{2^{\left[\log _{2} \frac{1}{u}\right]}}-u\right)+\phi\left(\frac{1}{2^{\left[\log _{2} \frac{1}{u}\right]}}\right)\left(\left(2^{\left[\log _{2} \frac{1}{u}\right]} u\right)^{p}-\left(1-2^{\left[\log _{2} \frac{1}{u}\right]} u\right)^{p}\right) .
$$

Proof. For $\left.u \in] 0, \frac{1}{2}\right]$, denote $\ell(u):=\left[\log _{2} \frac{1}{u}\right]$. Then by the definition of the integerpart function, we have $\ell(u)-1 \leq \log _{2} \frac{1}{u}-1<\ell(u)$; hence

$$
2^{\ell(u)-1} u \leq \frac{1}{2}<2^{\ell(u)} u \text {. }
$$

Thus, we have

$$
d_{\mathbb{Z}}\left(2^{n} u\right)= \begin{cases}2^{n} u, & \text { if } \quad n \leq \ell(u)-1 \\ d_{\mathbb{Z}}\left(2^{n}\left(\frac{1}{2^{\ell(u)}}-u\right)\right), & \text { if } \quad n \geq \ell(u)\end{cases}
$$


It can also be seen that

$$
d_{\mathbb{Z}}\left(2^{n}\left(\frac{1}{2^{\ell(u)}}-u\right)\right)=2^{n}\left(\frac{1}{2^{\ell(u)}}-u\right), \quad \text { if } \quad n \leq \ell(u) .
$$

Using these formulas and applying (13), we get

$$
\begin{aligned}
& \Phi_{p}(u)= \sum_{n=0}^{\ell(u)-2} \phi\left(\frac{1}{2^{n+1}}\right)\left(2^{p}\left(2^{n} u\right)^{p}-\left(2^{n+1} u\right)^{p}\right)+\phi\left(\frac{1}{2^{\ell(u)}}\right)\left(2^{p}\left(2^{\ell(u)-1} u\right)^{p}-\left(1-2^{\ell(u)} u\right)^{p}\right) \\
&+\sum_{n=\ell(u)}^{\infty} \phi\left(\frac{1}{2^{n+1}}\right)\left(2^{p} d_{\mathbb{Z}}^{p}\left(2^{n}\left(\frac{1}{2^{\ell(u)}}-u\right)\right)-d_{\mathbb{Z}}^{p}\left(2^{n+1}\left(\frac{1}{2^{\ell(u)}}-u\right)\right)\right) \\
&= \phi\left(\frac{1}{2^{\ell(u)}}\right)\left(\left(2^{\ell(u)} u\right)^{p}-\left(1-2^{\ell(u)} u\right)^{p}\right) \\
& \quad+\sum_{n=\ell(u)}^{\infty} \phi\left(\frac{1}{2^{n+1}}\right)\left(2^{p} d_{\mathbb{Z}}^{p}\left(2^{n}\left(\frac{1}{2^{\ell(u)}}-u\right)\right)-d_{\mathbb{Z}}^{p}\left(2^{n+1}\left(\frac{1}{2^{\ell(u)}}-u\right)\right)\right) \\
&=\phi\left(\frac{1}{2^{\ell(u)}}\right)\left(\left(2^{\ell(u)} u\right)^{p}-\left(1-2^{\ell(u)} u\right)^{p}\right) \\
& \quad+\sum_{n=0}^{\infty} \phi\left(\frac{1}{2^{n+1}}\right)\left(2^{p} d_{\mathbb{Z}}^{p}\left(2^{n}\left(\frac{1}{2^{\ell(u)}}-u\right)\right)-d_{\mathbb{Z}}^{p}\left(2^{n+1}\left(\frac{1}{2^{\ell(u)}}-u\right)\right)\right) \\
&=\phi\left(\frac{1}{2^{\ell(u)}}\right)\left(\left(2^{\ell(u)} u\right)^{p}-\left(1-2^{\ell(u)} u\right)^{p}\right)+\Phi_{p}\left(\frac{1}{2^{\ell(u)}}-u\right),
\end{aligned}
$$

which means that (14) holds.

In the next proposition an important class of functions $\phi$ from $\mathcal{H}$ will be described.

Proposition 6. Let $\phi:\left[0, \frac{1}{2}\right] \rightarrow \mathbb{R}_{+}$. Assume that $\phi(0)=0$ and the mapping $x \mapsto \frac{\phi(x)}{x}$ is concave on $\left.] 0, \frac{1}{2}\right]$. Then $\left.\phi\right|_{P} \in \mathcal{H}$, the function $x \mapsto \frac{\phi(x)}{x^{2}}$ is decreasing on $\left.] 0, \frac{1}{2}\right]$ and $\phi$ is continuous on $\left[0, \frac{1}{2}[\right.$.

Proof. By the concavity of the function $x \mapsto \frac{\phi(x)}{x}=: \psi(x)$, there exists an affine function that majorizes $\psi$; hence $\psi$ is bounded from above by a constant $C \geq 0$. Therefore,

$$
\left.\sum_{n=1}^{\infty} \phi\right|_{P}\left(\frac{1}{2^{n}}\right)=\sum_{n=1}^{\infty} \phi\left(\frac{1}{2^{n}}\right)=\sum_{n=1}^{\infty} \frac{1}{2^{n}} \psi\left(\frac{1}{2^{n}}\right) \leq C \sum_{n=1}^{\infty} \frac{1}{2^{n}}=C,
$$

which proves that (10) holds, i.e., $\left.\phi\right|_{P} \in \mathcal{H}$.

Let $0<z<y<x \leq \frac{1}{2}$. By the concavity of $x \mapsto \frac{\phi(x)}{x}$, we get

$$
\frac{\phi(y)}{y} \geq \frac{x-y}{x-z} \cdot \frac{\phi(z)}{z}+\frac{y-z}{x-z} \cdot \frac{\phi(x)}{x} .
$$

Since $\phi$ is nonnegative, by (15), we have that

$$
\frac{\phi(y)}{y} \geq \frac{y-z}{x-z} \cdot \frac{\phi(x)}{x} .
$$

Then, taking the limit $z \rightarrow 0$, we obtain $\frac{\phi(y)}{y^{2}} \geq \frac{\phi(x)}{x^{2}}$, which means that the mapping $x \mapsto \frac{\phi(x)}{x^{2}}$ is decreasing. 
Rearranging the inequality in (15), we get

$$
\phi(z) \leq z\left(\frac{\phi(y)}{y} \cdot \frac{x-z}{x-y}-\frac{\phi(x)}{x} \cdot \frac{y-z}{x-y}\right) .
$$

Upon taking the limit $z \rightarrow 0$ again, we get that $\phi$ is continuous at 0 . By the concavity assumption, it follows that $\phi$ is also continuous on $] 0, \frac{1}{2}[$.

In the next result we show that if $\phi$ is a cone combination of power functions, then $x \mapsto \frac{\phi(x)}{x}$ is concave.

Proposition 7. Let $\mu$ be a nonnegative bounded Borel measure on [1,2]. Let the error function $\phi_{\mu}: \mathbb{R}_{+} \rightarrow \mathbb{R}_{+}$be given by

$$
\phi_{\mu}(x):=\int_{1}^{2} x^{p} d \mu(p) \quad\left(x \in \mathbb{R}_{+}\right) .
$$

Then $x \mapsto \frac{\phi_{\mu}(x)}{x}$ is concave on $] 0,+\infty[$.

Proof. Define $\left.\psi_{\mu}:\right] 0,+\infty\left[\rightarrow \mathbb{R}_{+}\right.$by

$$
\psi_{\mu}(x):=\frac{\phi_{\mu}(x)}{x}=\int_{1}^{2} x^{p-1} d \mu(p) .
$$

Applying standard calculus rules, we have

$$
\psi_{\mu}^{\prime \prime}(x)=\int_{1}^{2}(p-1)(p-2) x^{p-3} d \mu(p),
$$

for all $x \in] 0,+\infty[$. By the nonnegativity of the measure and the nonpositivity of the integrand, we get that $\psi_{\mu}^{\prime \prime}(x) \leq 0$, if $x>0$. This implies that $\psi_{\mu}$ is concave on ] $0,+\infty[$, which completes the proof.

The next theorem has an important role in the proof of our subsequent main results.

Theorem 8. Let $\phi:\left[0, \frac{1}{2}\right] \rightarrow \mathbb{R}_{+}$. Assume that $\phi(0)=0$ and the mapping $x \mapsto \frac{\phi(x)}{x}$ is concave on $\left.] 0, \frac{1}{2}\right]$. Then, for all $u \in \mathbb{R}$,

$$
\Phi_{2}(u) \leq \phi \circ d_{\mathbb{Z}}(u) .
$$

Proof. By the 1-periodicity of $\Phi_{2}$, in the proof of (17), we may assume that $|u| \leq \frac{1}{2}$, and since $\Phi_{2}$ is even, we may also assume that $u>0$. We show, for every fixed $k \in \mathbb{N}$, that the theorem holds for all $u \in \mathbb{Z} / 2^{k}$. Then the statement follows from the continuity of $\Phi_{2}$ and $\phi$ and the denseness of dyadic rational numbers in $\mathbb{R}$.

Using induction on $m$, we prove that (17) holds for all $u=\frac{m}{2^{k}}$, where $m \in$ $\left\{1, \ldots, 2^{k-1}\right\}$. First we consider the case when $m=1$. Then, by (13),

$$
\begin{aligned}
\Phi_{2}\left(\frac{1}{2^{k}}\right) & =\sum_{n=0}^{k-1} \phi\left(\frac{1}{2^{n+1}}\right)\left(4 d_{\mathbb{Z}}^{2}\left(\frac{2^{n}}{2^{k}}\right)-d_{\mathbb{Z}}^{2}\left(\frac{2^{n+1}}{2^{k}}\right)\right) \\
& =\sum_{n=0}^{k-2} \phi\left(\frac{1}{2^{n+1}}\right)\left(4\left(\frac{4^{n}}{4^{k}}\right)-\frac{4^{n+1}}{4^{k}}\right)+\phi\left(\frac{1}{2^{k}}\right)=\phi\left(\frac{1}{2^{k}}\right),
\end{aligned}
$$

which shows that in this case (17) holds with equality. 
Now let $m \in\left\{2, \ldots, 2^{k-1}\right\}$ and assume that (17) has been verified for all $u=\frac{j}{2^{k}}$, where $j \in\{1, \ldots, m-1\}$. With the notation $\ell(u):=\left[\log _{2} \frac{1}{u}\right]$, it is easy to see that $\frac{1}{2^{\ell(u)}}-u \in \mathbb{Z} / 2^{k}$ and $0<\frac{1}{2^{\ell(u)}}-u<u$; thus by the inductive assumption, we have

$$
\Phi_{2}\left(\frac{1}{2^{\ell(u)}}-u\right) \leq \phi\left(\frac{1}{2^{\ell(u)}}-u\right) .
$$

In view of Proposition 5 with $p=2$, the identity

$$
\Phi_{2}(u)=\Phi_{2}\left(\frac{1}{2^{\ell(u)}}-u\right)+\phi\left(\frac{1}{2^{\ell(u)}}\right)\left(2^{\ell(u)+1} u-1\right)
$$

holds. By (19) and (18) we have that

$$
\Phi_{2}(u) \leq \phi\left(\frac{1}{2^{\ell(u)}}-u\right)+\phi\left(\frac{1}{2^{\ell(u)}}\right)\left(2^{\ell(u)+1} u-1\right) .
$$

Thus to prove the assertion of the theorem it is enough to show that

$$
\phi\left(\frac{1}{2^{\ell(u)}}-u\right)+\phi\left(\frac{1}{2^{\ell(u)}}\right)\left(2^{\ell(u)+1} u-1\right) \leq \phi(u) .
$$

Let $\left.\left.\psi(x)=\frac{\phi(x)}{x}(x \in] 0, \frac{1}{2}\right]\right)$. Then, by the concavity of $\psi$, we get

$$
\begin{aligned}
\left(\frac{1}{2^{\ell(u)} u}-1\right) \psi\left(\frac{1}{2^{\ell(u)}}-u\right) & +\left(2-\frac{1}{2^{\ell(u)} u}\right) \psi\left(\frac{1}{2^{\ell(u)}}\right) \\
& \leq \psi\left(\left(\frac{1}{2^{\ell(u)}}-u\right)\left(\frac{1}{2^{\ell(u)} u}-1\right)+\frac{1}{2^{\ell(u)}}\left(2-\frac{1}{2^{\ell(u)} u}\right)\right)=\psi(u),
\end{aligned}
$$

which is equivalent to (20), completing the proof of (17).

The main result of this paper is stated in the following theorem.

Theorem 9. Let $\phi:\left[0, \frac{1}{2}\right] \rightarrow \mathbb{R}_{+}$. Assume that $\phi(0)=0$ and the mapping $x \mapsto \frac{\phi(x)}{x}$ is concave on $\left.] 0, \frac{1}{2}\right]$. Then $S_{\phi}$ is approximately Jensen convex in the following sense: For all $x, y \in \mathbb{R}$,

$$
S_{\phi}\left(\frac{x+y}{2}\right) \leq \frac{S_{\phi}(x)+S_{\phi}(y)}{2}+\phi \circ d_{\mathbb{Z}}\left(\frac{x-y}{2}\right) .
$$

Proof. For an arbitrary function $f: \mathbb{R} \rightarrow \mathbb{R}$ define $J f: \mathbb{R}^{2} \rightarrow \mathbb{R}$ as in the proof of Theorem 4. By Proposition [6] we have that $\left.\phi\right|_{P} \in \mathcal{H}$, which, by Lemma 1, implies that $S_{\phi}$ is well-defined. By Proposition 6, we get that $x \mapsto \frac{\phi(x)}{x^{2}}$ is decreasing, which means, for all $\left.u \in] 0, \frac{1}{2}\right]$, that

$$
\phi(2 u) \leq 4 \phi(u)
$$

This means that we can apply Theorem 4 with $p=2$ for the function $S_{\phi}$. Thus we obtain that for all $x, y \in \mathbb{R}$,

$$
J S_{\phi}(x, y) \leq \Phi_{2}\left(\frac{x-y}{2}\right) .
$$

In view of Theorem 8, we also have (17) for all $u \in \mathbb{R}$. Therefore, (22) and (17) imply that (21) holds for all $x, y \in \mathbb{R}$, which means that $S_{\phi}$ is approximately Jensen convex in the sense of (21).

The next result immediately follows from Proposition 7 and the above theorem. The particular case when $\phi$ is of the form $\phi(t)=\varepsilon t^{p}$ (where $\varepsilon \geq 0$ and $p \in[1,2]$ are constants) was discovered by Tabor and Tabor in [22] and was already stated in Theorem 4 .

Corollary 10. Let $\mu$ be a nonnegative bounded Borel measure on $[1,2]$. Let the error function $\phi_{\mu}: \mathbb{R}_{+} \rightarrow \mathbb{R}_{+}$be given by (16). Then, for all $x, y \in \mathbb{R}$,

$$
S_{\phi_{\mu}}\left(\frac{x+y}{2}\right) \leq \frac{S_{\phi_{\mu}}(x)+S_{\phi_{\mu}}(y)}{2}+\phi_{\mu} \circ d_{\mathbb{Z}}\left(\frac{x-y}{2}\right) .
$$




\section{Applications to $\varphi$-CONVEXity}

We shall prove that the error term $\mathcal{S}_{\varphi}(t,|x-y|)$ in (44) under certain assumptions on the error function $\varphi$ is the smallest possible one. In other words, the next theorem will provide an exact upper bound for the convexity-difference of $\varphi$-Jensen convex functions defined by (8).

Theorem 11. Let $\varphi: \frac{1}{2} I^{*} \rightarrow \mathbb{R}$ be nondecreasing and assume that $\varphi(0)=0$ and $x \mapsto \frac{\phi(x)}{x}$ is concave on $\frac{1}{2} I^{*}$. Then, for all $x, y \in I$ and $t \in[0,1]$,

$$
C_{\varphi}(x, y, t)=\mathcal{S}_{\varphi}(t,|x-y|) ;
$$

furthermore, for fixed $x, y \in I$, the function $f_{x, y}: I \rightarrow \mathbb{R}$ defined by

$$
f_{x, y}(u)=\mathcal{S}_{\varphi}\left(\frac{y-u}{y-x},|x-y|\right) \quad(u \in I)
$$

is $\varphi$-Jensen convex on $I$ and, for all $t \in[0,1]$,

$$
f_{x, y}(t x+(1-t) y)-t f_{x, y}(x)-(1-t) f_{x, y}(y)=\mathcal{S}_{\varphi}(t,|x-y|) .
$$

Proof. From the concavity of $x \mapsto \frac{\varphi(x)}{x}$ it follows (as in Proposition 6) that, for some $n_{0} \in \mathbb{N}$, the series $\sum_{n=n_{0}}^{\infty} \varphi\left(2^{-n}\right)$ is convergent. Hence, by Theorem $\mathbb{B}$ we get that

$$
C_{\varphi}(x, y, t) \leq \mathcal{S}_{\varphi}(t,|x-y|)
$$

for all $x, y \in I$ and $t \in[0,1]$.

Now let $x, y \in I$ be fixed. In order to show the reversed inequality (which then yields (23) $)$, it suffices to prove that the function $f_{x, y}$ defined in (24) is $\varphi$-Jensen convex on $I$ and (25) holds for all $t \in[0,1]$.

Observe that $f_{x, y}(x)=f_{x, y}(y)=0$. Hence, by putting $u=t x+(1-t) y$ in (24), the equality (25) follows. by

To verify that $f_{x, y}$ is $\varphi$-Jensen convex on $I$, define the function $\phi:\left[0, \frac{1}{2}\right] \rightarrow \mathbb{R}_{+}$

$$
\phi(r):=\varphi(r|x-y|) .
$$

Then, we get that $r \mapsto \frac{\phi(r)}{r}$ is concave on $\left.] 0, \frac{1}{2}\right]$. Therefore, by Theorem 9 , the Takagi type function $S_{\phi}$ satisfies

$$
S_{\phi}\left(\frac{s+t}{2}\right) \leq \frac{S_{\phi}(s)+S_{\phi}(t)}{2}+\phi \circ d_{\mathbb{Z}}\left(\frac{s-t}{2}\right)
$$

for all $t, s \in \mathbb{R}$. Observe that, for all $t \in \mathbb{R}$,

$$
S_{\phi}(t)=\sum_{n=0}^{\infty} 2 \phi\left(\frac{1}{2^{n+1}}\right) d_{\mathbb{Z}}\left(2^{n} t\right)=\sum_{n=0}^{\infty} 2 \varphi\left(\frac{|x-y|}{2^{n+1}}\right) d_{\mathbb{Z}}\left(2^{n} t\right)=\mathcal{S}_{\varphi}(t,|x-y|) .
$$

On the other hand, using the increasingness of $\phi$, for $s, t \in \frac{1}{x-y}(I-y)$, we have

$$
\phi\left(d_{\mathbb{Z}}\left(\frac{s-t}{2}\right)\right) \leq \phi\left(\frac{|s-t|}{2}\right)=\varphi\left(\frac{|s-t|}{2}|x-y|\right) .
$$

Thus, for $s, t \in \frac{1}{x-y}(I-y)$, (27) can be rewritten as

$$
\mathcal{S}_{\varphi}\left(\frac{s+t}{2},|x-y|\right) \leq \frac{\mathcal{S}_{\varphi}(s,|x-y|)+\mathcal{S}_{\varphi}(t,|x-y|)}{2}+\varphi\left(\frac{|s-t|}{2}|x-y|\right) .
$$


Taking $u, v \in I$ arbitrarily, the values $s=\frac{y-u}{y-x}$ and $t=\frac{y-v}{y-x}$ belong to $\frac{1}{x-y}(I-y)$ and hence (28) reduces to

$$
f_{x, y}\left(\frac{u+v}{2}\right) \leq \frac{f_{x, y}(u)+f_{x, y}(v)}{2}+\varphi\left(\frac{|u-v|}{2}\right),
$$

which completes the proof of the $\varphi$-Jensen convexity of $f_{x, y}$.

Taking an error function $\varphi$ which is a combination of power functions of exponents from $[1,2]$, we obtain the following result.

Theorem 12. Let $I$ be a nonempty real interval and $\mu$ be a nonnegative bounded Borel measure on $[1,2]$. Define the error function $\varphi_{\mu}: \mathbb{R}_{+} \rightarrow \mathbb{R}_{+}$by (16). Then, for all $x, y \in I$ and $t \in[0,1]$,

$$
C_{\varphi_{\mu}}(x, y, t)=\int_{1}^{2} S_{p}(t)|x-y|^{p} d \mu(p)
$$

where $S_{p}: \mathbb{R} \rightarrow \mathbb{R}$ is given by (5).

Proof. It is easy to see that $\varphi_{\mu}$ is nondecreasing and we also have that $\varphi_{\mu}(0)=0$.

By Proposition 7 we get that $u \mapsto \frac{\varphi_{\mu}(u)}{u}$ is concave on ]0, $+\infty$ [. Thus, Theorem 11 can be applied, and hence, for all $x, y \in I$ and $t \in[0,1]$,

$$
C_{\varphi_{\mu}}(x, y, t)=\mathcal{S}_{\varphi_{\mu}}(t,|x-y|) .
$$

On the other hand, for all $x, y \in I$ and $t \in[0,1]$,

$$
\begin{aligned}
\mathcal{S}_{\varphi_{\mu}}(t,|x-y|) & =\sum_{n=0}^{\infty} 2 \varphi_{\mu}\left(\frac{|x-y|}{2^{n+1}}\right) d_{\mathbb{Z}}\left(2^{n} t\right)=\sum_{n=0}^{\infty} \int_{1}^{2} 2\left(\frac{|x-y|}{2^{n+1}}\right)^{p} d \mu(p) d_{\mathbb{Z}}\left(2^{n} t\right) \\
& =\int_{1}^{2} \sum_{n=0}^{\infty} \frac{1}{2^{n+p-1}} d_{\mathbb{Z}}\left(2^{n} t\right)|x-y|^{p} d \mu(p)=\int_{1}^{2} S_{p}(t)|x-y|^{p} d \mu(p),
\end{aligned}
$$

which completes the proof.

Taking $\mu$ as the point measure $\varepsilon \delta_{p}$ where $p \in[1,2]$ in the above theorem, it reduces to a result by Jacek and Józef Tabor [22], which states that (7) is sharp, i.e., that the term $\varepsilon S_{p}(t)$ is the smallest possible.

Corollary 13. Let $I$ be a nonempty real interval, $p \in[1,2]$ and $\varepsilon \geq 0$. Define the error function $\varphi: \mathbb{R}_{+} \rightarrow \mathbb{R}_{+}$by $\varphi(u):=\varepsilon u^{p}$. Then, for all $x, y \in I$ and $t \in[0,1]$,

$$
C_{\varphi}(x, y, t)=\varepsilon S_{p}(t)|x-y|^{p} .
$$

\section{REFERENCES}

[1] F. Bernstein and G. Doetsch, Zur Theorie der konvexen Funktionen, Math. Ann. 76 (1915), no. 4, 514-526. MR1511840

[2] P. Billingsley, Notes: Van Der Waerden's Continuous Nowhere Differentiable Function, Amer. Math. Monthly 89 (1982), no. 9, 691. MR.1540053

[3] Z. Boros, An inequality for the Takagi function, Math. Inequal. Appl. 11 (2008), no. 4, 757-765. MR2458169(2009f:39047)

[4] P. Cannarsa and C. Sinestrari, Semiconcave functions, Hamilton-Jacobi equations, and optimal control, Progress in Nonlinear Differential Equations and their Applications, 58, Birkhäuser Boston Inc., Boston, MA, 2004. MR2041617 (2005e:49001)

[5] F. S. Cater, On van der Waerden's nowhere differentiable function, Amer. Math. Monthly 91 (1984), no. 5, 307-308. MR740246 (85j:26009)

[6] D. H. Hyers and S. M. Ulam, Approximately convex functions, Proc. Amer. Math. Soc. 3 (1952), 821-828. MR0049962(14,254b) 
[7] A. Házy, On the stability of t-convex functions, Aequationes Math. 74 (2007), no. 3, 210-218. MR2376448(2008j:26012)

[8] A. Házy and Zs. Páles, On approximately midconvex functions, Bull. London Math. Soc. 36 (2004), no. 3, 339-350. MR2038721(2004j:26020)

[9] , On approximately t-convex functions, Publ. Math. Debrecen 66 (2005), 489-501. MR2137784 (2006c:26023)

[10] - On a certain stability of the Hermite-Hadamard inequality, Proc. R. Soc. Lond. Ser. A Math. Phys. Eng. Sci. 465 (2009), 571-583. MR2471774 (2009k:39033)

[11] H.-H. Kairies, Takagi's function and its functional equations, Rocznik Nauk.-Dydakt. Prace Mat. (1998), no. 15, 73-83. MR1826076 (2002b:39014)

[12] K. Knopp, Ein einfaches Verfahren zur Bildüng stetiger nirgends differenzierbarer Funktionen, Math. Z. 2 (1918), no. 1-2, 1-26. MR1544308

[13] J. Makó and Zs. Páles, Approximate convexity of Takagi type functions, J. Math. Anal. Appl. 369 (2010), 545-554. MR2651700 (2011k:26011)

[14] Strengthening of strong and approximate convexity, Acta Math. Hungar. 132 (2011), no. 1-2, 78-91. MR2805478

[15] A. Mureńko, Ja. Tabor, and Jó. Tabor, Applications of de Rham Theorem in approximate midconvexity, J. Diff. Equat. Appl. 18 (2012), no. 3, 335-344. MR2901825

[16] C. T. Ng and K. Nikodem, On approximately convex functions, Proc. Amer. Math. Soc. 118 (1993), no. 1, 103-108. MR.1159176 (93f:26006)

[17] Zs. Páles, On approximately convex functions, Proc. Amer. Math. Soc. 131 (2003), no. 1, 243-252. MR1929044 (2003h:26015)

[18] S. Rolewicz, On paraconvex multifunctions, Third Symposium on Operations Research (Univ. Mannheim, Mannheim, 1978), Section I, Operations Res. Verfahren, vol. 31, Hain, Königstein/Ts., 1979, pp. 539-546. MR0541221 (80i:49025)

[19] _ On $\gamma$-paraconvex multifunctions, Math. Japon. 24 (1979/80), no. 3, 293-300. MR0550213 (81a:52013)

[20] , Paraconvex analysis, Control Cybernet. 34 (2005), no. 3, 951-965. MR.2208979 (2006m:49025)

[21] Ja. Tabor and Jó. Tabor, Generalized approximate midconvexity, Control Cybernet. 38 (2009), no. 3, 655-669. MR2650358 (2011f:52002)

[22] , Takagi functions and approximate midconvexity, J. Math. Anal. Appl. 356 (2009), no. 2, 729-737. MR2524305(2010d:26017)

[23] Ja. Tabor, Jó. Tabor, and M. Żołdak, Approximately convex functions on topological vector spaces, Publ. Math. Debrecen 77 (2010), 115-123. MR2675738 (2011f:26009)

[24] Optimality estimations for approximately midconvex functions, Aequationes Math. 80 (2010), 227-237. MR2736954 (2011j:26020)

[25] T. Takagi, A simple example of the continuous function without derivative, J. Phys. Math. Soc. Japan 1 (1903), 176-177.

[26] B. L. van der Waerden, Ein einfaches Beispiel einer nichtdifferenzierbaren stetigen Funktion, Math. Z. 32 (1930), 474-475. MR1545179

Institute of Mathematics, University of Debrecen, Pf. 12, H-4010 Debrecen, Hungary E-mail address: makoj@science.unideb.hu

Institute of Mathematics, University of Debrecen, Pf. 12, H-4010 Debrecen, Hungary E-mail address: pales@science.unideb.hu 